\title{
Development process vacuum-thermal filling of polymers with lubricating material
}

\author{
Filippenko Nikolay \\ Transport University \\ Irkutsk, 664075, Russia \\ e-mail: ifpi@mail.ru \\ Bychkovsky Vladimir \\ Transport University \\ Irkutsk, 664075, Russia \\ e-mail: bikovskii_vs@mail.ru
}

\begin{abstract}
Under certain processing conditions, the relationship between the anodic current and the polymer material that was changed during the processing by electrophysical parameters was substantiated and determined on the basis of the equation of high-frequency heating. In the course of the review, the advantages and disadvantages of the existing oil-filling methods were determined, the goal and tasks of further research. This work determines the reasons that cause the integrity violations of the structure of polyamide materials in the process of their operation, based on the methods proposed and developed by the authors. The damage was investigated taking into account the real meteorological and climatic conditions of operation of the rolling stock of Russian Railways. Using the example of glass-filled polyamide samples, necessary and sufficient parameters for defining the strength characteristics of the investigated material were determined. Experimental relationships were obtained for determining the kinetics of the formation of fractures of polymer materials
\end{abstract}

Keywords-polymers, welding, heating processes, automation

\section{INTRODUCTION}

To increase the strength properties of polymers under conditions of depleted lubrication of mating parts (dry start), oil-filled polymer and composite materials are created. The addition of liquids, thermal plasticizers, partially filling the space between the polymer chains, facilitates their movement and flexibility.

Studies have shown that the realization of oil-filling technological processes raises the issue of ensuring selective heating of the filled polymer sample, does not affect the filler (oil) and vice versa.

Existing equipment for oil filling of polymeric and composite antifriction materials is distinguished by oil filling methods:

- adding oil directly to the polymer solution;

- aging in oil under various conditions.

\author{
Lipchitz Alexandr \\ Transport University \\ Irkutsk, 664075, Russia \\ e-mail: livnet@list.ru \\ Popov Maxim \\ Transport University \\ Irkutsk, 664075, Russia \\ e-mail: popovs@irgups.ru
}

The basis for osmosis is the generally known fact of hygroscopicity of most polymeric materials [1]. Some groups of polymers can absorb up to $10 \%$ moisture.

The experimental data on the hygroscopicity of polymers are shown in the graph (Fig.1)

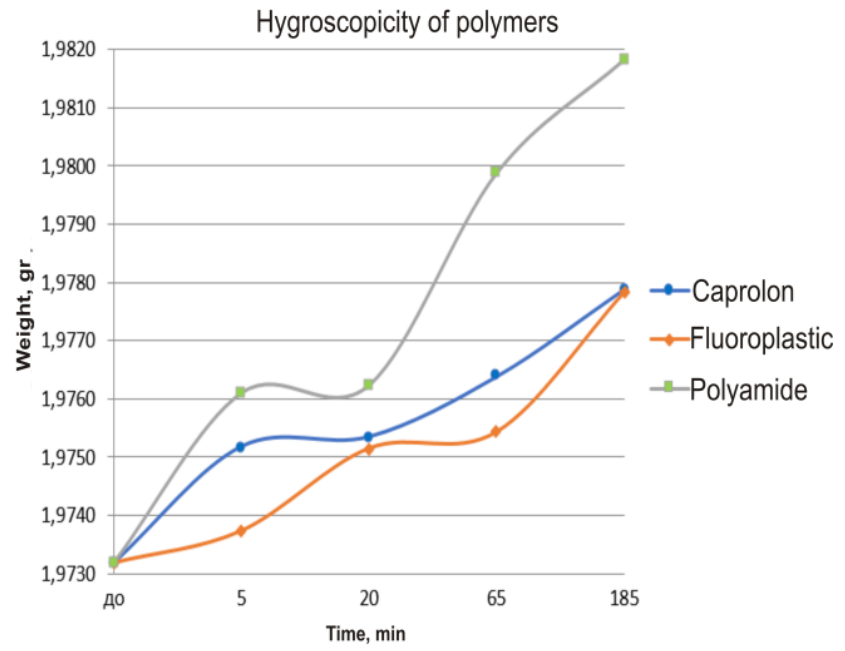

Fig. 1 Graph of hygroscopicity of polymeric and composite antifriction materials

\section{ADDING OIL DIRECTLY TO THE POLYMER SOLUTION}

One of the main and most common method of filling oil with a solution of polymers was developed by Japanese authors: Morino Katsuaki, Aoyama Teruo, Furuiti Minoru, Maeda Masaki, Okada Kouji, Koudzin Dzunji [2].

The authors of this methodology developed a new oilfilled 1, 2-polybutadiene having functions characteristic of conventional 1,2-polybutadiene with distinctive properties in terms of wear resistance, flowability (manufacturability), the ability to paint (high image accuracy), flexibility and ability to attach. As oil fillers, aromatic oil fillers, naphthenic oil fillers and paraffin oil fillers were used. 
Advantages of this technology are: uniformly distributed lubricant inside the material ensuring its constant operation during the entire service life of the product, reducing wear, improving frictional properties in abrasive wear, the lubricant does not dry out, is not removed during machining or friction, and does not need replenishment. However, with the introduction of oils, a "thin-layer" plasticization of the polymer occurs, leading to an increase in the elasticity and flowability of the polymer.

\section{FILLING IN THE FILLER UNDER NORMAL CONDITIONS}

Flash-technology of making printed forms and stamps. The most widely used technology is filling polymers directly onto the finished product, namely special rubber [3]. For flash technology, heat-sensitive porous rubber is needed. In the process of making a stamp or printed form, some pores of rubber are baked. On the baked surface, a stencil is applied from a special photosensitive carbon film. The light energy of the flash lamp is converted into thermal energy $\left(70-75^{\circ} \mathrm{C}\right)$ and creates a printing surface of the positive image on the rubber. When the light of a lamp is absorbed, the carbon film heats up and, on contact with the surface of the rubber, bakes its pores. The pores under the image remain open, which are used for filling with printing ink.

It takes 1-2 hours to completely saturate the rubber, but if the colorant is heated to a temperature of $30-35^{\circ} \mathrm{C}$, the filling time can be reduced several times. The time of saturation of a mold or stamp is determined empirically.

The manufacturing process includes 5 stages:

1. Output of the original layout (positive);

2. Installation of the workpiece in a flash unit;

3. Backlight;

4. Mounting the cliche in the snap;

\section{Filling with paint}

Stamps made with the help of flash technology have a number of advantages. There are no protruding parts on the printing surface that can wear out and crumble, you do not need a constant ink supply. The number of impressions obtained after one refueling can be 5-8 thousand, after which the form can be refilled. It is possible to obtain multicolored stamps, that is, to use simultaneously different coloring substances, differing in both chemical and physical composition (different sizes of the pigment) and electrothermal (HF treatment).

\section{AUTOMATED LIQUID-PHASE FILLING OF POLYMERS AND COMPOSITES BASED ON THEM WITH ENGINE OILS}

The authors of [4] based on flash technology developed the technology of liquid-phase filling polymers and composites on their basis with engine oils. It presented the results of studies of liquid-phase filling of polymers and composites based on engine oils and the goal they set was to obtain materials with increased wear resistance and load capacity.

To carry out experimental studies on thermovacuum oil filling of polymers and composite antifriction materials, the authors modernized the experimental setup, based on the industrial equipment of the model AZ pre-ink N2, intended for the production of ink-filled flash cliches. The scheme of the experimental setup is shown in Fig. 2.

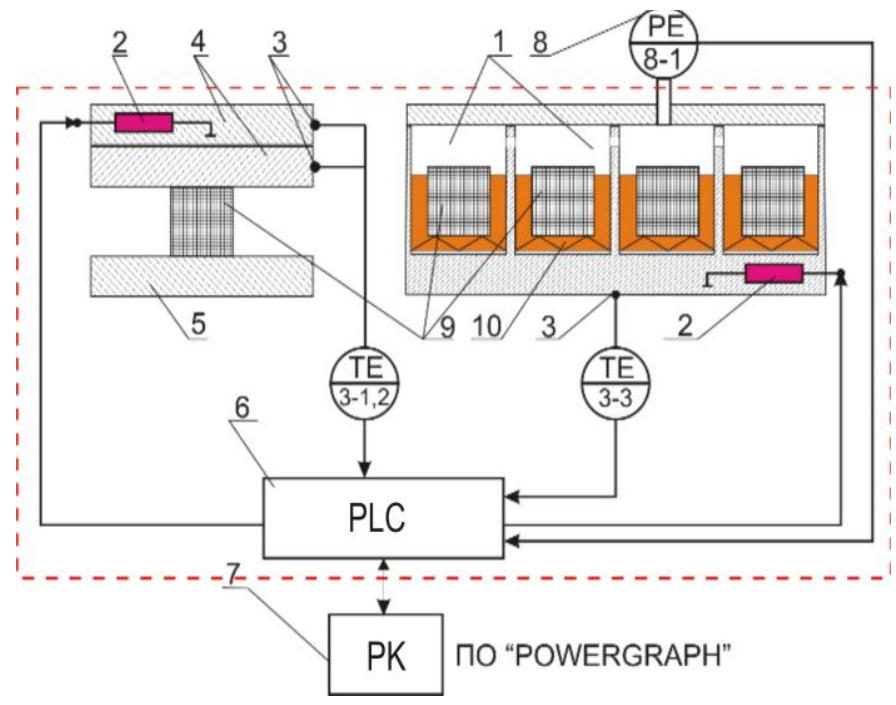

Fig. 2 Automated experimental setup: 1 - vacuum chamber, 2 - heater; 3 thermocouple; 4 - installation plates; 5 - press plate; 6 - microcontroller; 7 computer; 8 - gauge of vacuum; 9 - sample; 10 - antifriction liquid

But it should be noted that this technology does not allow the flow of oil filler from the open pores of the material to be avoided during the transportation or storage of products in the warehouse. Also, the thermovacuum method of heating the polymer material does not guarantee uniform and selective heating of the product.

\section{HEATING OF POLYMERIC MATERIALS AND HEATING OF THE OIL FILLER IN ORDER TO REDUCE ITS VISCOSITY BY HF TREATMENT}

For heating and drying, electrical oscillations of industrial frequency (inductive heating), radio waves of high and ultrahigh frequencies (dielectric heating) and infrared radiation (infrared heating) are mainly used.

It is known that the most effective method of heating polymers is dielectric heating. In HF drying of polymers, the main driving force of moisture is the excessive pressure that is formed when the same moisture evaporates.

It should be noted that in the process of electrothermal processing, the system of the HF generator-technological device is affected by a number of external and internal factors that do not allow the operating mode to remain constant. Here are the main of them [1]:

a) articles, blanks from polymeric materials are, as a rule, a geometrically complex structure, and the composition of polymers has a multicomponent structure. Changes in the electrophysical parameters of products in the process of transportation, storage, operation can reach several percent;

b) in the process of HF exposure, the electrophysical parameters of the material vary with the frequency of the EMW and the temperature [5, 6], which corresponds to the expression (1): 


$$
\varepsilon^{\prime \prime}=F(f),
$$

where $\varepsilon^{\prime \prime}=\varepsilon^{\prime} \cdot \operatorname{tg} \delta$ is the dielectric loss factor;

c) in the process of increasing the load of the HF generator, its frequency characteristics change $\pm 0.6 \%$ [7];

d) in the process of the supply of electrical energy, the GOST sets the quality standards, the indicators of which can vary up to $10 \%$, and the indicator for the voltage failure time can reach 30 seconds. [7], which is comparable to the full cycle of HF processing;

e) the melting point (transition to the viscous state) for polymeric materials is not a constant value $[8,9,10]$.

Nevertheless, in [1], the interconnection of the anodic current with the polymer material that was changed during the processing by electrophysical parameters was substantiated and determined on the basis of the equation of high-frequency heating (2):

$$
I_{a}=\frac{4 \times \pi \times \varepsilon_{o} \times \varepsilon^{\prime \prime}(T) \times E \times U}{\gamma \times d^{2}},
$$

where $\varepsilon_{0}$ is the dielectric constant;

$E$ is the electric field strength;

$U$ - voltage on the capacitor plates;

$\gamma$ is the conductivity;

$d$ is the distance between the capacitor plates.

The author concluded that the change in the physicochemical (phase) state of the processed material can be traced to the dynamics of the change in the dielectric loss factor. Hence, the control of the electrophysical parameters of the polymer material in the process of HF treatment can be justified by the single parameter of the operation of the electrothermal equipment-the anode current Ia in Fig. 3.

The analysis of the obtained data showed characteristic changes in the anode current as a function of the temperature of the sample under study. Analysis of graph 4 [11] shows that the change in the phase state of the samples (the beginning of melting) corresponds to the first minimum of the values of the anode current.

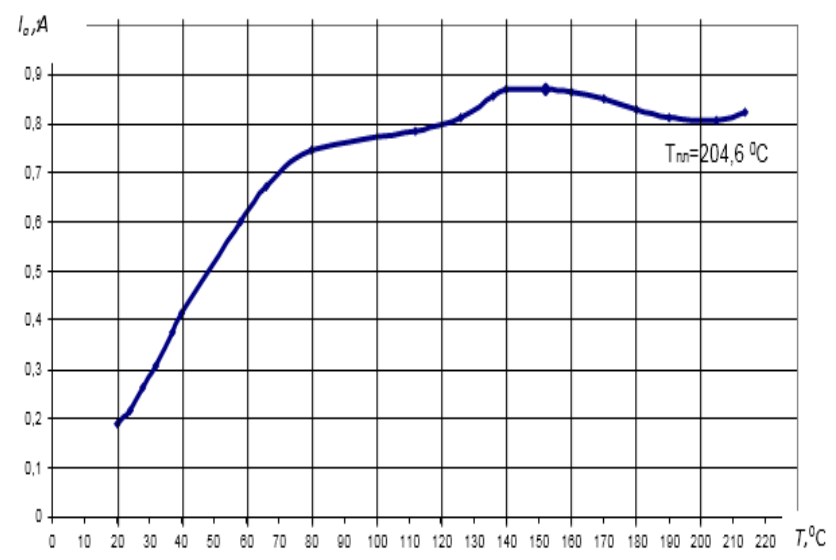

Fig. 3. Amperometric dependence of the phase transformation of polyamide
In the course of the study, a technique for controlling $\mathrm{HF}$ processing of polymer materials was developed. As was pointed out [11], the only controlled parameter of $\mathrm{HF}$ processing is the initial phase transition moment, which is also true for the HF drying process.

According to the adopted decision, the entire technological process of HF processing was presented in the form of a set of the following stages of drying:

1. The stage of "acclimatization," characterized by a smooth increase in the intensity of the HF field, is accompanied by the burning out of foreign surface inclusions.

2. The stage of reaching the beginning of the "thawing of segmental bonds" corresponding to the achievement of the first peak of the anode current and a temperature corresponding to approximately $90 \%$ of $\mathrm{Tm}$.

3. Stabilization of the HF-drying process by $90 \%$ of $\mathrm{Tf}$ (cyclic regulation in the automatic mode of power of impact), with continuous monitoring of the development of breakdown.

4. At the end of the drying time, the processing is stopped.

The author [11] developed a method for controlling the electrothermal process:

- preparatory treatment of the product directly in the process of HF exposure (to further intensify the processing process);

- determination of the phase state of the polymer being processed (by the method of comparative analysis of the instantaneous changes in the anode current);

- long-term definition of the prebreakdown condition, by the intensity (time of occurrence) of partial discharges;

- organization of the regulatory process, based on the conditions of maximum energy efficiency.

The universality of this technique lies in the fact that for the HF process (heating, drying, melting) it is applicable to any type of equipment and products made of polymer materials of any geometric shape and design.

The developed electrothermal process control technique is acceptable for further investigation of oil filling of products made of polymer materials by electrothermal heating..

\section{CONCLUSION}

According to the review of patent and literature sources, it was found that there are basically two methods of oil-filling polymers (adding oil to the solution and aging in oil under special conditions).

It was also determined that:

- the addition of oil to the solution of the polymeric material has basically the following advantages: the lubricant is uniformly distributed inside the material ensuring its constant work during the entire service life of the product; reduces wear; Improving frictional properties in abrasive wear, the lubricant does not dry out, is not removed during machining or friction, and never needs replenishment. The 
main disadvantage of this method is the increase in the elasticity and flowability of the polymer;

- aging in oil under normal conditions has the following positive qualities: increased wear resistance and load capacity; capable of ensuring the operation of friction units in the absence of lubrication; reduction of the coefficient of friction. The presence of negative qualities indicates that this oil-filling effect is short-lived, since only a relatively small amount of liquid lubricant can be introduced into the polymer without complicating the osmosis technology, and in the process of transporting or storing products in the warehouse, the possibility of the oil filler outflow from the open pores of the material is high.

A method for heating a polymer material with selective heating over a part or lubricant by means of HF heating was also determined, and a controlling parameter for electrothermal heating was set.

Based on the foregoing, the goal of further research was the development of the technology of electrothermal oil filling of parts made of polymer materials, the achievement of which is possible after solving a number of problems:

- determine the types of lubricants, depending on the operating conditions;

- to choose a technique of oil filling of products from polymeric materials with various geometry;

- develop a method for eliminating leakage of filler from oil-filled polymer parts;

- to develop the technology of electrothermal oil filling of polymer materials.

The results of the work will be published after further research.

\section{References}

[1] GOST 10589-87 “Castablepolyamide 610”, Moscow: Publishing Standards, 1987, $12 \mathrm{p}$.

[2] GOST 22372-77. "Dielectric Materials. Methods for determining the dielectric permittivity and tangent of the dielectric loss angle", Moscow: Izd-vostandartov Publ., 1977, 12 p.

[3] D.G. Gromakovsky, V.P. Efimov, E.P. Kovtunov, E.P. Mokhonko, G.A. Kulakov, I.D. Ibatullin "Tribotechnical foundations for increasing the wear resistance of rails, wheels and wheel bearings", [International scientific andtechnical conference Actual problems of reliability of technological, power and transport machines proceedings]. Samara: Mashinostroenie Publ., 2003, pp. 207-212.

[4] D.G. Gromakovsky, I.D. Ibatullin "A new method for evaluating the plasticity of materials”, August 2003. pp. 51-56 [Seminar Acoustic Emission Diagnostic Method for Railway Transport. Bookof reports St. Petersburg: RP Association of developers and manufacturers of scienceintensive products for railways, 2003]

[5] F.R. Gekker, S.I. Khayramiev "Influence of the dynamic contact interaction on the sliding friction", Machine Science. 1985, pp. 88-93.

[6] O.I. Boginsky. Strengthening the movement safety.Wagons and rolling stock. St. Petersburg, No.4 (12) M.2007, pp. 10-13.

[7] A.V. Livshits, A.Y. Mashovich. N.G. Filippenko, "Aspects of electrothermal processing of materials by an electromagnetic field of high frequency", Modern technologies.System analysis. Modeling. 2011. Issue 2. (30). pp.135-140.

[8] V.M. Finkel, "Physical basis of deceleration decay", Moscow: Metallurgiya Publ, 1977, 360 p.

[9] V.A. Likhachev, V.G. Malinin, "Structural-analytic theory of strength", St. Petersburg: Nauka Publ., 1995. Vol.1. 298 p., Vol.2. 320 p.

[10] V.A. Zakrevsky, V.A. Okhotin "Autoionization mechanism of rupture of chemical bonds in macromolecules. High-molecular bonds", 1981. Vol. 23A. No. 3. pp. 658-667.

[11] N.G. Filippenko "Mathematical model of the process of high-frequency processing of polymeric materials", Modern technologies.System analysis. Modeling. 2012. Issue 1 (33). pp. 76 - 79. 